BMJ Open

Diabetes

Research

\& Care

\title{
Central administration of sodium- glucose cotransporter-2 inhibitors increases food intake involving adenosine monophosphate-activated protein kinase phosphorylation in the lateral hypothalamus in healthy rats
}

Kenji Takeda (D), Hiraku Ono (D), Ko Ishikawa (D), Tomohiro Ohno, Jin Kumagai, Hidetoshi Ochiai, Ai Matumoto, Hidetaka Yokoh, Yoshiro Maezawa (D), Koutaro Yokote

To cite: Takeda $\mathrm{K}, \mathrm{Ono} \mathrm{H}$, Ishikawa K, et al. Central administration of sodiumglucose cotransporter-2 inhibitors increases food intake involving adenosine monophosphate-activated protein kinase phosphorylation in the lateral hypothalamus in healthy rats. $B M J$ Open Diab Res Care 2021;9:e002104. doi:10.1136/ bmjdrc-2020-002104

- Supplemental material is published online only. To view please visit the journal online (http://dx.doi.org/10.1136/ bmjdrc-2020-002104).

Received 31 December 2020 Revised 3 March 2021 Accepted 21 March 2021

Check for updates

\section{(c) Author(s) (or their} employer(s)) 2021. Re-use permitted under CC BY-NC. No commercial re-use. See rights and permissions. Published by BMJ.

Department of Endocrinology, Hematology, and Gerontology, Chiba University Graduate School of Medicine, Chiba, Japan

Correspondence to Dr Hiraku Ono; hono@chiba-u.jp

\section{ABSTRACT}

Introduction Sodium glucose cotransporter-2 (SGLT2) inhibitors are widely used for diabetes treatment. Although SGLT2 inhibitors have been clinically observed to increase food intake, roles or even the presence of SGLT2 in the central nervous system (CNS) has not been established. We aimed to elucidate potential functions of SGLT2 in the CNS, and the effects of CNS-targeted SGLT2 inhibitors on food intake.

Research design and methods We administered three kinds of SGLT2 inhibitors, tofogliflozin, dapagliflozin, and empagliflozin, into the lateral ventricle (LV) in rats and evaluated their effects on food intake. We also evaluated the effects of tofogliflozin administration in the third (3V) and fourth ventricle (4V). Intraperitoneal administration of liraglutide, a glucagonlike peptide-1 (GLP-1) receptor agonist known to suppress food intake, was combined with central tofogliflozin to elucidate whether GLP-1 signaling antagonizes the effect of central SGLT2 inhibitors on food intake. To elucidate potential molecular mechanisms mediating changes in feeding, hypothalamic areas associated with food intake regulation were harvested and analyzed after intracerebroventricular administration (ICV) of tofogliflozin.

Results Bolus ICV injection of tofogliflozin induced a robust increase in food intake starting at 1.5 hours postinjection, and lasting for 5 days. No effect was observed when the same dose of tofogliflozin was administered intraperitoneally. ICV dapagliflozin and empagliflozin significantly enhanced food intake, although the strength of these effects varied among drugs. Food intake was most markedly enhanced when tofogliflozin was infused into the LV. Fewer or no effects were observed with infusion into the $3 \mathrm{~V}$ or $4 \mathrm{~V}$, respectively. Systemic administration of liraglutide suppressed the effect of ICV tofogliflozin on food intake. ICV tofogliflozin increased phosphorylation of AMPK and c-fos expression in the lateral hypothalamus.

Conclusions SGLT2 inhibitors in the CNS increase food intake. SGLT2 activity in the CNS may regulate food intake through AMPK phosphorylation in the lateral hypothalamic area.

\section{Significance of this study}

What is already known about this subject?

- Intracerebroventricular administration of phlorizin, a non-specific sodium glucose cotransporter (SGLT) inhibitor, increases food intake in rats.

- Systemic clinical use of SGLT2 inhibitors in humans increases food intake.

What are the new findings?

- Administration of SGLT2 inhibitors within the central nervous system (CNS) increases food intake in rats.

- Systemic administration of liraglutide suppresses the effect of intracerebroventricular administrated tofogliflozin on food intake.

- Inhibition of SGLT2 within the CNS enhances AMPK phosphorylation in the lateral hypothalamic area.

How might these results change the focus of research or clinical practice?

- SGLT2 in the CNS is suggested to be involved in regulating food intake.

- SGLT2 inhibitors with low penetration of the blood brain barrier could be used to treat diabetes with less compensatory food intake.

\section{INTRODUCTION}

Glucose, the main source of energy in the brain, is a large polar molecule that cannot pass through the lipid bilayer of cell membranes by simple diffusion. Hence, cells have glucose transporters to take up glucose. ${ }^{1}$ Three families of solute carriers have been identified as glucose transporters: GLUTs (glucose transporters), SGLTs (sodiumglucose cotransporters), and SWEETs (sugars will eventually be exported transporters). ${ }^{2}$ GLUTs transport glucose across the plasma 
membrane via facilitated diffusion, while SGLTs use the sodium concentration gradient generated by the sodium-potassium ATPase as a source of potential energy to symport glucose in concert with sodium ions. ${ }^{3} \mathrm{Six}$ SGLT proteins have been identified in humans. SGLT1/ SLC5A1 and SGLT2/SLC5A2 are expressed in the kidney and play an important role in reabsorption of glucose from raw urine. ${ }^{2}$ SGLT2 inhibitors contribute to reducing blood glucose levels by inhibiting reabsorption of glucose from raw urine. Although urinary glucose elimination results in both calorie loss and weight loss, the actual weight loss observed with clinical use is reportedly smaller than the expected weight loss due to glucose discharge. ${ }^{4}$ The appetite-stimulating effects of SGLT2 inhibitors in clinical use are of concern, but the mechanism by which these inhibitors stimulate food intake via the central nervous system (CNS) is not yet fully understood. ${ }^{4}$

SGLT2/SLC5A2 is reportedly expressed specifically on brush border membranes of proximal tubular epithelial cells in the kidney. ${ }^{5-7}$ At the mRNA level, conflicting reports exist regarding its expression in the CNS. ${ }^{5-9}$ Thus, the presence of SGLT2/SLC5A2 in the CNS is controversial. Based on western blotting results, SGLT2/SLC5A2 protein is present in human brain tissue after trauma. ${ }^{10}$ Furthermore, SGLT2/ SLC5A2 has been detected in choroid plexus epithelial cells and ependymal cells in normal human and mouse tissues by immunostaining. ${ }^{11}$ Another recent report showed that SGLT2/SLC5A2 is present in the forebrains and brain stems of mice. ${ }^{12}$ One study suggests that glucose uptake occurs via SGLT2/SLC5A2 in the rat brain. ${ }^{13}$ To date, expression of SGLT2/SLC5A2 in the rat brain has not been unequivocally proven, and its function in the CNS thus remains uncertain. ${ }^{11}$

Phlorizin, which inhibits both SGLT1/SLC5A1 and SGLT2/SCL5A2, increased food intake in rats when administered into the cerebral ventricle. ${ }^{14-17}$ 2-deoxy-D-glucose (2-DG) is a non-metabolizable glucose analogue ${ }^{18}$ which, when taken into cells, antagonizes normal intracellular glucose metabolism, ${ }^{19}$ but stimulates food intake when injected into the cerebral ventricles of rats. ${ }^{152}$ Impaired glucose metabolism in the brain stimulates food intake. ${ }^{15}$ Considering that clinical use of SGLT2 inhibitors is known to induce food intake, ${ }^{4}$ CNS-expressed SGLT2/SLC5A2 may play a role in regulating food intake. There remains a possibility that prescribed SGLT2 inhibitors can cross the blood brain barrier (BBB) and act in brain regions that govern appetite and satiety. ${ }^{821}$

If SGLT2/SLC5A2 is present in the CNS in rats as well as in humans and mice, then intracerebroventricular administration (ICV) infusion of an SGLT2 inhibitor, like phlorizin, might increase food intake. In this study, we aimed to elucidate whether SGLT2/SLC5A2 in the CNS plays a role in stimulating food intake.

\section{RESEARCH DESIGN AND METHODS}

\section{Animals and surgical treatment}

Male Sprague-Dawley rats (CLEA Japan, Tokyo, Japan) weighing 250-350 g were housed separately in air-conditioned animal quarters with lights on between 06:00 and 18:00 hours. Food and water were available ad libitum throughout the study. After 7 days of adaptation to their home cages, rats were anaesthetized with isoflurane (Mylan, Pennsylvania, USA). A 23-gauge stainless steel cannula (P1 Technologies, Virginia, USA) was stably implanted into the ventricle using a stereotaxic apparatus. Right lateral ventricle (LV) coordinates were $0.8 \mathrm{~mm}$ posterior, $1.4 \mathrm{~mm}$ right lateral, and $4.3 \mathrm{~mm}$ ventral to the bregma. Third ventricle (3V) coordinates were $3.0 \mathrm{~mm}$ posterior and $9.7 \mathrm{~mm}$ ventral to the bregma, and fourth ventricle $(4 \mathrm{~V})$ coordinates were $12.0 \mathrm{~mm}$ posterior and $9.0 \mathrm{~mm}$ ventral to the bregma, in accordance with the atlas. ${ }^{22}$ The cannula was secured to the skull with three stainless steel screws and dental cement. The implanted cannula was temporarily plugged with a 33-gauge stainless dummy cannula. After surgery, the animals were allowed 5 days to recover. Correct positioning of the cannula was verified by ICV infusion of angiotensin II (100 ng, Sigma-Aldrich, Missouri, USA) to confirm a dipsogenic response. All experiments were performed more than a week after the surgery. After the experiments, $3 \mu \mathrm{L}$ of black ink was injected into the cannula of each animal, and cannula placement was confirmed by spreading of the dye throughout the cerebral ventricular system.

\section{Materials}

2-DG (20 $\mu$ mol, Merck KGaA, Darmstadt, DE, Germany), phlorizin $(0.52 \mu \mathrm{mol}$, Merck KGaA), tofogliflozin (0.52 umol, Advanced ChemBlocks, California, USA), empagliflozin $(0.52 \mu \mathrm{mol}$, ChemScene, New Jersey, USA), and dapagliflozin $(0.52$ or $4.16 \mu \mathrm{mol}$, Carbosynth, Berks, UK) were dissolved in vehicle, consisting of $25 \%$ DMSO (dimethyl sulfoxide) in $75 \%$ normal saline, and then administered ICV to the rats. DMSO (25\%) was required to dissolve each solute. The glucagon-like peptide-1 (GLP-1) receptor agonist, liraglutide (20 $\mu \mathrm{g}$, Adipogen, California, USA) was dissolved in vehicle, consisting of $200 \mu \mathrm{L}$ of saline containing $2 \%$ DMSO, and injected intraperitoneally. For quantitative PCR, total RNA was prepared from tissue using an RNeasy Plus Mini Kit (Qiagen, Hilden, DE, Germany). Complementary DNA was prepared using a PrimeScript RT Reagent kit (Takara, Shiga, Japan). TaqMan probes for c-fos, agoutirelated protein (AgRP), neuropeptide $\mathrm{Y}(\mathrm{NPY})$, and proopiomelanocortin (POMC) were obtained from Thermo Fisher Scientific (Rn02396759_m, Rn01431703_g1, Rn00561681_m1, and Rn00595020_m1, respectively; MA, USA) and were used with TaqMan Fast Advanced Master Mix (Applied Biosystems, California, USA). 18s primers (18S-f: CGGACAGGATTGACAGATTG, 18S-r: CAAATCGCTCCACCAACTAA) were purchased from Applied Biosystems and used with Fast SYBR Green Master Mix in a CFX Connect Real-Time PCR Detection System (Bio-Rad, California, USA). For western blotting, frozen tissues were sonicated in lysis buffer containing a phosphatase inhibitor cocktail (F. Hoffmann-La Roche, Basel, CH, Switzerland) as previously described. ${ }^{23}$ c-fos 
antibody (sc-271243, 1:100) was purchased from Santa Cruz Biotechnology (California, USA). Phospho-AMPK $\alpha$ (Thr172) antibody (\#2531 1:1000), AMPK $\alpha$ (\#2532 1:1000) antibody, $\beta$-tubulin (9F3) rabbit mAb (\#2128 1:1000), HRP-linked anti-mouse IgG (\#7072 1: 5000), and HRP-linked anti-rabbit IgG (\#7074 1:1000) were from Cell Signaling Technology (MA, USA). Blocking was performed with $0.5 \%$ bovine serum albumin or $1 \%$ skim milk. Western Lightning Plus-ECL was obtained from Perkin Elmer (Massachusetts, USA).

\section{ICV infusion}

Experiments were begun between 09:00 and 12:00 hours. ICV infusion was performed for 3 min using a motordriven infusion pump with a delivery rate set to $1 \mu \mathrm{L} /$ min. The rats were awake during the ICV procedure. Starting 2 days before the day of drug administration, and for up to 6 days thereafter, food intake, water intake, and body weight were measured daily. Cumulative food intake, water intake, and body weight were measured at $0,1.5,3,6$, and 24 hours after completion of infusion. Spilled food was accounted for in each measurement.

\section{Administration of tofogliflozin and liraglutide}

The time and method of administration were the same as those applied for ICV infusion. Tofogliflozin was administered only once, while liraglutide was administered daily at 09:00 hours during the observation period. The measurement method was the same as that used for ICV infusion.

\section{Quantitative PCR and western blotting}

More than a week after the surgery, tofogliflozin, 2-DG, or vehicle was infused as described above. Animals were decapitated 1.5 hours after drug administration. Brain tissues were frozen in liquid nitrogen and stored at $-80^{\circ} \mathrm{C}$. Each brain was cut into serial $2.5 \mathrm{~mm}$ sections for micro-punch dissection of the paraventricular nucleus (PVH, 0.0 to $-2.5 \mathrm{~mm}$ ), the arcuate nucleus (ARC, -2.5 to $-5.0 \mathrm{~mm}$ ), the lateral hypothalamus ( $\mathrm{LH},-2.5$ to $-5.0 \mathrm{~mm}$ ), and the ventromedial hypothalamic nucleus (VMH, -2.5 to $-5.0 \mathrm{~mm}$ ) spanning indicated neuroanatomical intervals relative to the anterior commissure. ${ }^{22}$ Tissue samples were procured with a biopsy punch (diameter: $1 \mathrm{~mm}$, Integra Life Science, New Jersey, USA). For western blotting, after tissue sonication, protein concentrations in lysis buffer were measured and normalized. Proteins were blotted with the indicated antibodies. Band intensities from western blots were measured and quantified using Image J software.

\section{Data analysis}

All statistical analyses were performed using GraphPad Prism 8 (V.8.4.3). Data for each drug treatment protocol were evaluated by one-way analysis of variance (ANOVA) for each phenotype. Where indicated, further comparisons between individual groups and the vehicle group were performed using Dunnett's multiple comparison test. Data obtained for multiple days were analyzed by two-way or three-way repeated measures ANOVA. In some analyses, variances among groups differed significantly using the F-test but were not significantly different after logarithmic transformation. In these analyses, ANOVA and multiple comparisons were performed using logarithmically transformed values. Unless otherwise noted, data are presented as the mean $\pm \mathrm{SD}$. A significant difference was defined as $\mathrm{p}<0.05$.

\section{RESULTS}

\section{Administration of tofogliflozin into the LV increased food} intake

To investigate whether CNS administration of SGLT2 inhibitors affects food intake, we administered 0.52 $\mu \mathrm{mol} / 3 \mu \mathrm{L}$ of tofogliflozin, $0.52 \mu \mathrm{mol} / 3 \mu \mathrm{L}$ of phlorizin, $20 \mu \mathrm{mol} / 3 \mu \mathrm{L}$ of $2-\mathrm{DG}$, or $3 \mu \mathrm{L}$ of vehicle into the $\mathrm{LV}$ via stably implanted cannula. We chose the same molar amount of $0.52 \mu \mathrm{mol}$ SGLT2 inhibitors as was used in a previous report in which phlorizin increased food intake. ${ }^{17}$ We decided that $20 \mu \mathrm{mol}$ of 2-DG (in accord with a previous report) would be sufficient to increase food intake. ${ }^{15}$ Tofogliflozin induced a significant increase in food intake at 1.5, 3, 6, and 24 hours after bolus administration, compared with vehicle (figure 1A). In a preliminary experiment using another cohort of rats, the food intake at 24 hours did not reach statistical significance ( $\mathrm{p}=0.10$; data not shown). Consistent with previous reports, increased food intake was also observed with ICV infusions of phlorizin and 2-DG. Increased food intake was only observed during the first 1.5 hours after ICV phlorizin administration (figure 1A). Tofogliflozin tended to yield stronger effects on food intake than 2-DG and phlorizin. Tofogliflozin also induced a significant increase in water intake 1.5, 3, 6, and 24 hours after bolus administration, compared with vehicle (figure 1B). In accordance with the increased food intake, rats administered tofogliflozin gained significantly more body weight than those administered vehicle alone. The difference in weight gain was approximately equal to the sum of the increased food and water intake (figure 1C). With phlorizin and 2-DG, the increase in food intake disappeared after 24 hours. Increased food and water intake with tofogliflozin administration persisted for 5 days. (figure 1D,E). Weight gain with tofogliflozin did not persist beyond the day after administration.

\section{Systemic administration of tofogliflozin did not change food intake}

To confirm that the effects of ICV tofogliflozin on food intake are exerted directly on the CNS and not via systemic effects after leakage out of the brain, we administered the same molar amount of tofogliflozin intraperitoneally, and observed no significant effects on food or water intake, or body weight in response to systemic tofogliflozin administration (online supplemental figure 1).

\section{ICV administration of other SGLT2 inhibitors also increased food intake}

To examine whether the effects on food intake induced by ICV tofogliflozin are compound-specific or common 

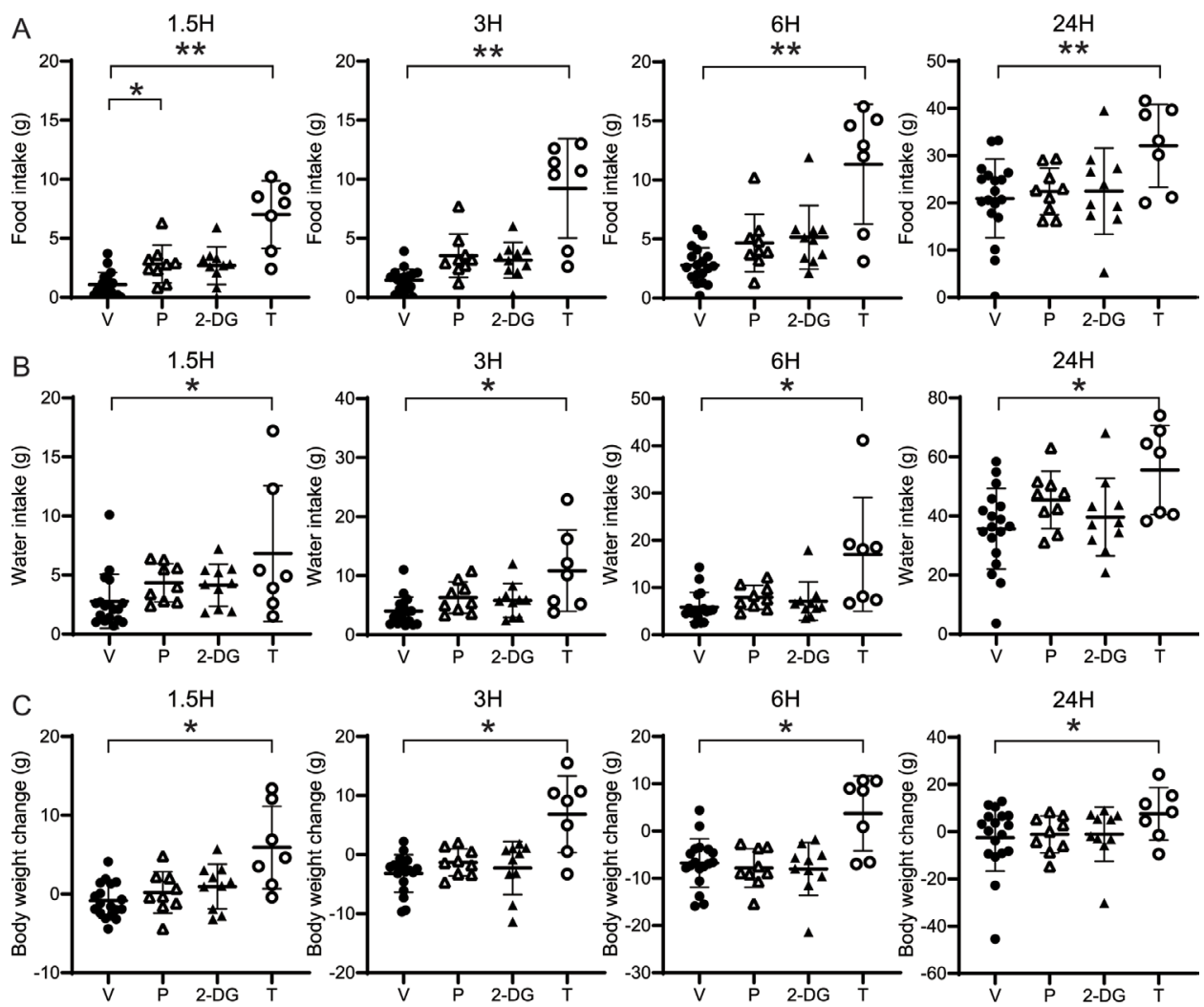

$\mathrm{D}$

$\mathrm{E}$
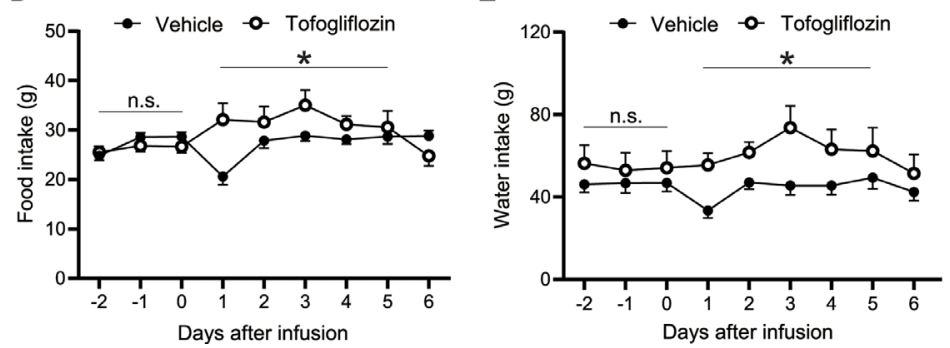

Figure 1 Effects of ICV tofogliflozin on food intake, water intake and body weight. $0.52 \mu \mathrm{mol} / 3 \mu \mathrm{L}$ of tofogliflozin, 0.52 $\mu \mathrm{mol} / 3 \mu \mathrm{L}$ of phlorizin, $20 \mu \mathrm{mol} / 3 \mu \mathrm{L}$ of $2-\mathrm{DG}$, or vehicle was infused into the LV of rats, and food and water intake and body weight gain were measured at 1.5, 3, 6, and 24 hours after completion of infusion, and from 2 days before, to 6 days after ICV infusion. (A) Cumulative food intake after ICV administration. (B) Cumulative water intake after ICV administration. (C) Body weight change relative to weight at time of drug administration. (D) Daily food intake from 2 days before, to 6 days after administration. (E) Daily water intake from 2 days before to 6 days after administration. (A-C) Data are presented as the mean $\pm S D$. $(D, E)$ Data are presented as the mean $\pm S E M$. Significance relative to vehicle control: ${ }^{*} p<0.05,{ }^{* *} p<0.01$. V: vehicle, P: phlorizin, 2-DG: 2-deoxy-D-glucose, T: tofogliflozin A-E (V, P, 2-DG, T) n=18, 9, 10, and 7, respectively. ICV, intracerebroventricular administration; LV, lateral ventricle.

to SGLT2 inhibitors, two other SGLT2 inhibitors, dapagliflozin and empagliflozin, were administered into the LV using the same protocol. As shown in figure 2A, dapagliflozin significantly increased food intake at 6 hours. ICV empagliflozin significantly enhanced food intake at 1.5 hours (figure 2B). Water intake and body weight did not change significantly over the course of the experiment in response to either of these drugs.

To determine whether higher doses of SGLT2 inhibitors would induce more robust effects on food intake, $4.16 \mu \mathrm{mol}$ (eightfold higher concentration than in the previous experiment) dapagliflozin was administered into the LV. Compared with vehicle, high-dose dapagliflozin significantly increased food intake at 1.5, 3, and
6 hours, and water intake at 6 and 24 hours (figure 2C,D). There was no significant change in body weight. Food and water intake were significantly elevated by high-dose dapagliflozin for 5 days (figure 2E,F).

\section{The hyperphagic effect induced by ICV tofogliflozin was the strongest in the LV, followed by the $3 \mathrm{~V}$}

To investigate where in the brain SGLT2 inhibitors acts to enhance feeding, tofogliflozin was administered into the $3 \mathrm{~V}$ and $4 \mathrm{~V}$. Administration of $0.52 \mu \mathrm{mol}$ tofogliflozin into the 3 V increased food intake at 1.5 and 3 hours (figure $3 \mathrm{~A}$ ). A trend toward increased food intake was still present at 6 hours, although it did not reach statistical significance. Regardless of the increase in food intake, no significant 
A

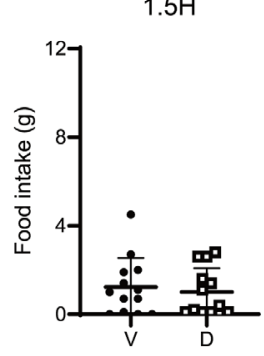

B

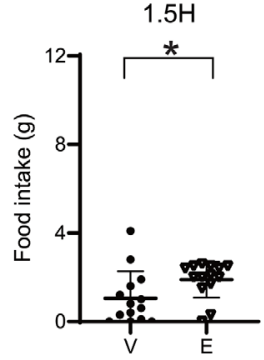

C

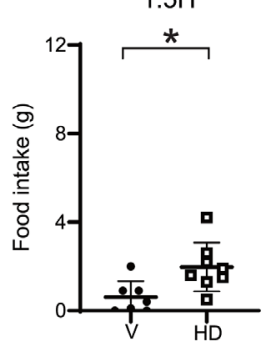

D

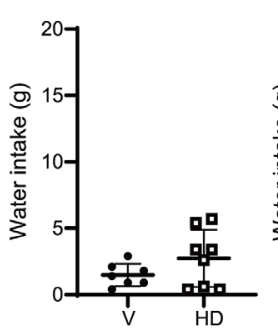

E
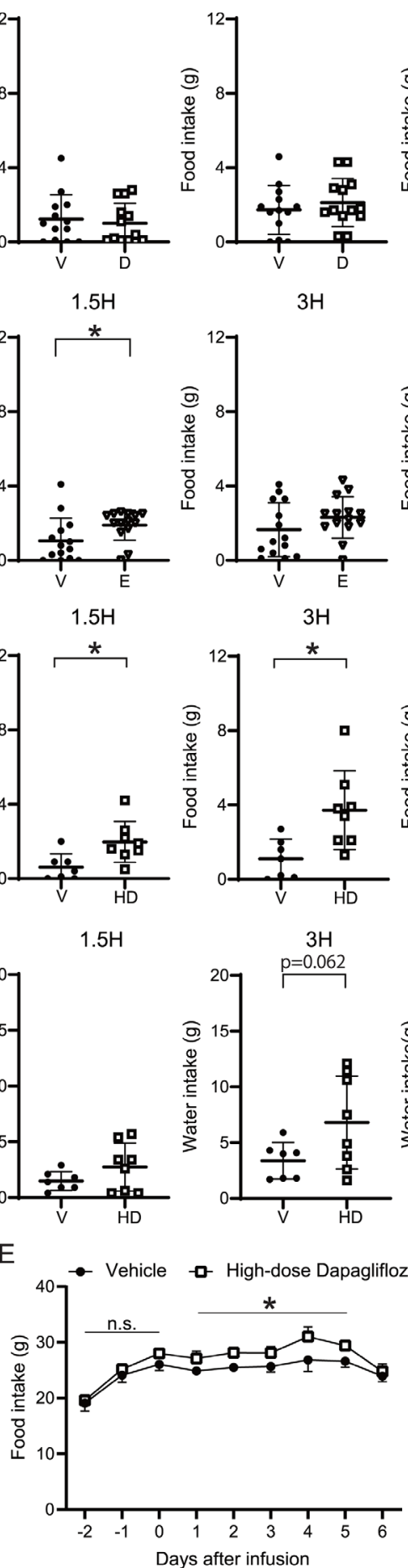

$3 \mathrm{H}$

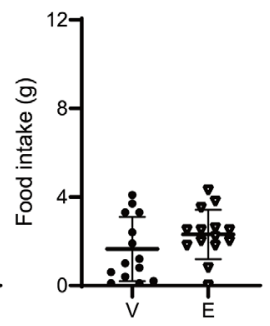

$3 \mathrm{H}$

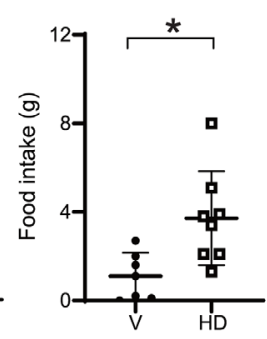

$3 \mathrm{H}$

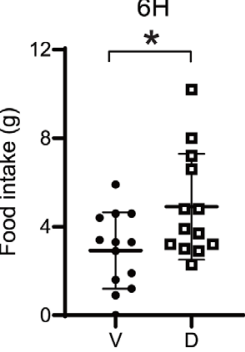

$6 \mathrm{H}$

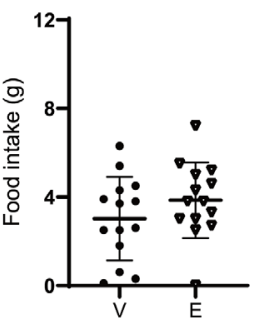

$6 \mathrm{H}$
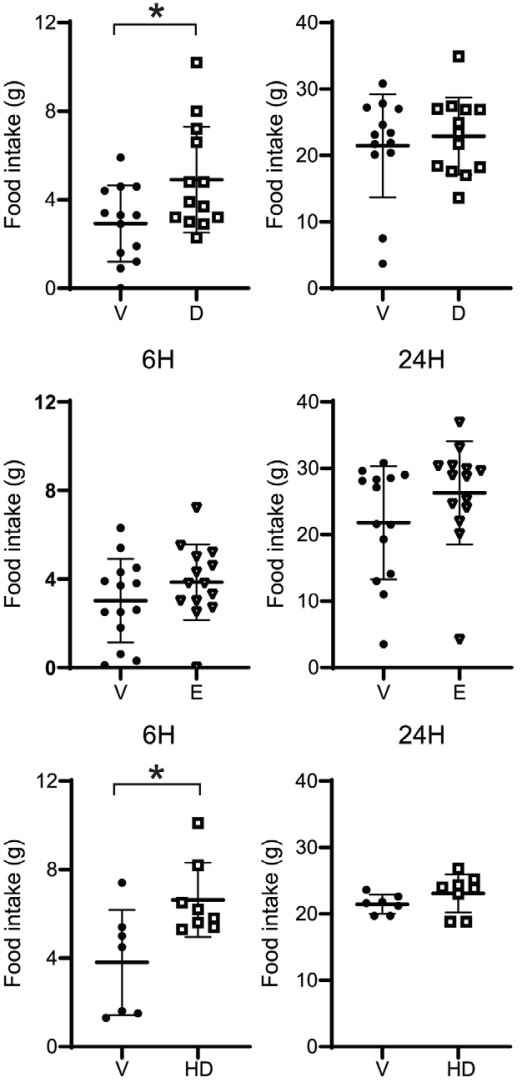

$24 \mathrm{H}$

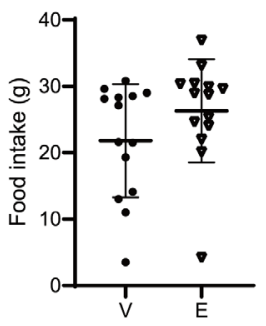

$24 \mathrm{H}$
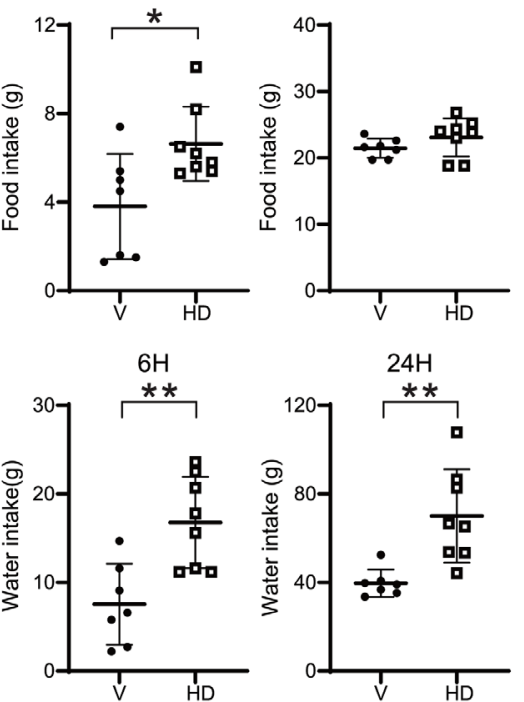

$F$

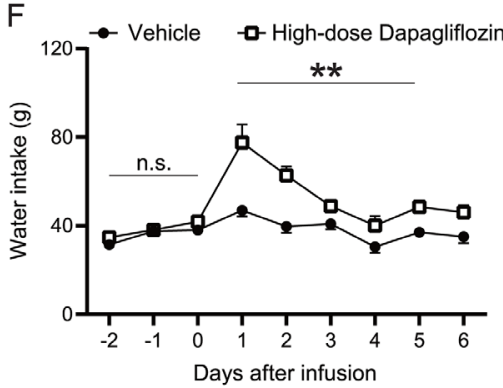

Figure 2 Effects of ICV infusion of dapagliflozin and empagliflozin on food intake and water intake. $0.52 \mu \mathrm{mol}$ or $4.16 \mu \mathrm{mol}$ (high-dose) $/ 3 \mu \mathrm{L}$ of dapagliflozin, $0.52 \mu \mathrm{mol} / 3 \mu \mathrm{L}$ of empagliflozin or $3 \mu \mathrm{L}$ of vehicle was infused into the $\mathrm{LV}$ of rats, and food and water intake were measured at 0, 1.5, 3, 6, and 24 hours after ICV infusion. (A) Cumulative food intake after ICV 0.52 $\mu \mathrm{mol}$ dapagliflozin administration. (B) Cumulative food intake after ICV $0.52 \mu \mathrm{mol}$ empagliflozin administration. (C) Cumulative food intake. (D) Cumulative water intake after high-dose dapagliflozin administration. (E) Daily food and (F) water intake from 2 days before to 6 days after high-dose dapagliflozin administration. (A-D) Data are presented as the mean \pm SD. (E,F) Data are presented as the mean \pm SEM. Significance relative to vehicle control: ${ }^{*} p<0.05,{ }^{* *} p<0.01$. V: vehicle, $D$ : dapagliflozin, $E$ : empagliflozin, HD: high-dose dapagliflozin $A(V, D): n=13$ and 13 , respectively. $B(V, E): n=14$ and 14 , respectively. C-F (V, HD) $\mathrm{n}=7$ and 8 , respectively. ICV, intracerebroventricular administration; LV, lateral ventricle. 
A

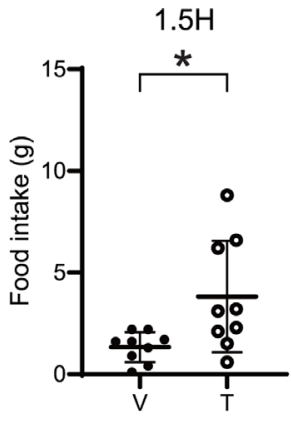

B
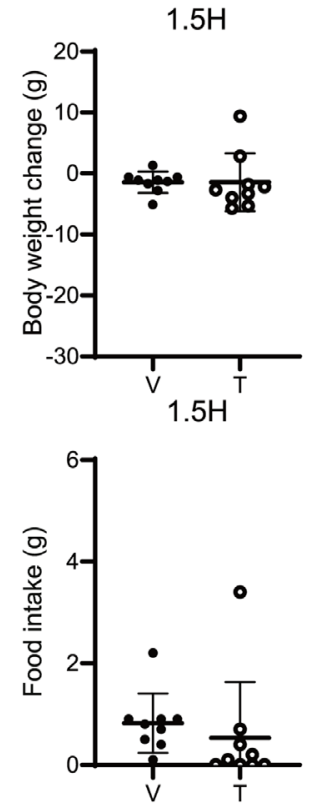

D

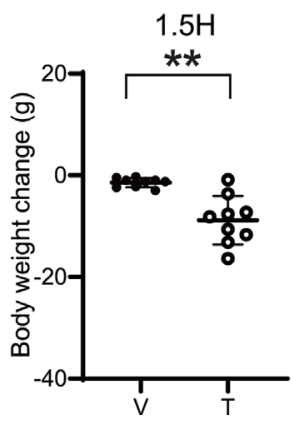

$3 \mathrm{H}$

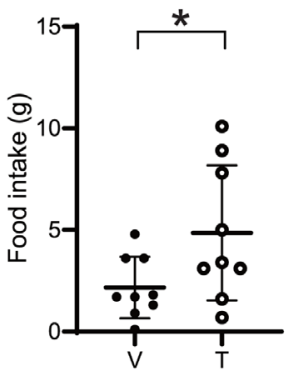

$3 \mathrm{H}$
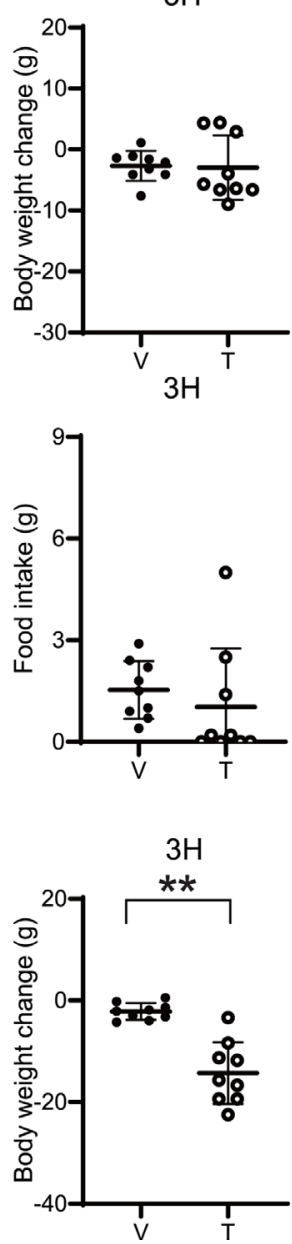

$6 \mathrm{H}$

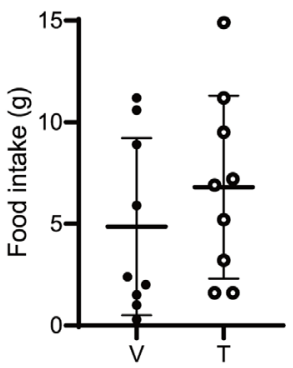

$6 \mathrm{H}$
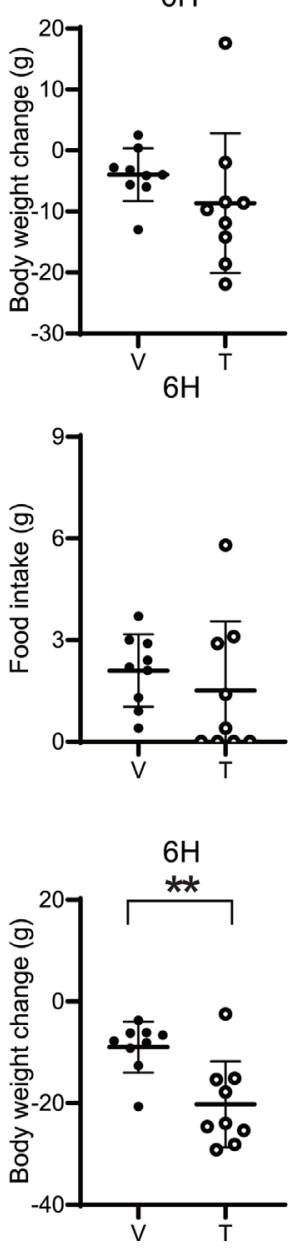
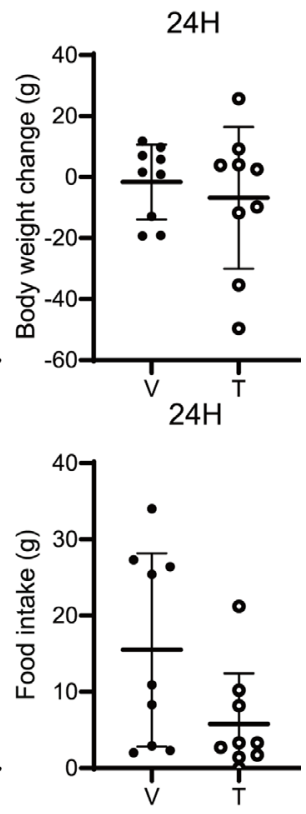

$24 \mathrm{H}$

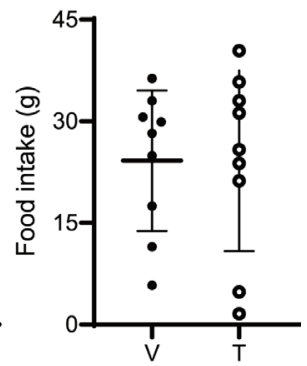

$24 \mathrm{H}$

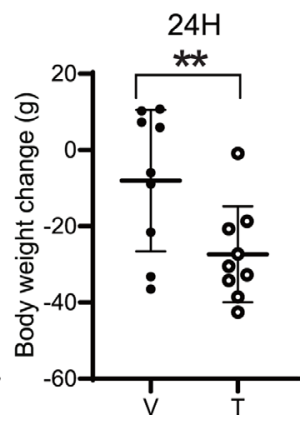

Figure 3 Effects of ICV infusion of tofogliflozin into $3 \mathrm{~V}$ or $4 \mathrm{~V}$ on food intake and body weight. $0.52 \mu \mathrm{mol} / 3 \mu \mathrm{L}$ of tofogliflozin or vehicle was infused into the $3 \mathrm{~V}$ or $4 \mathrm{~V}$ of rats, and food intake and body weight gain were measured at 1.5, 3, 6 , and 24 hours after completion of infusion, and from 2 days before to 6 days after. (A) Cumulative food intake after ICV administration into the 3V. (B) Body weight change relative to the time of drug administration into the 3V. (C) Cumulative food intake after ICV administration into the 4V. (D) Body weight change relative to the time of drug administration into the $4 \mathrm{~V}$. Data are presented as the mean \pm SD. Significance relative to vehicle control: ${ }^{*} p<0.05$, ${ }^{* *} p<0.01$. V: vehicle, T: tofogliflozin $A-D(V, T): n=9$ and 9 , respectively. ICV, intracerebroventricular administration; $3 \mathrm{~V}$, third ventricle; $4 \mathrm{~V}$, fourth ventricle.

change in body weight was observed (figure 3B). Administration of tofogliflozin into the $4 \mathrm{~V}$ increased neither food nor water intake (figure 3C). Rather, food and water intake tended to decrease. Moreover, a significant decrease in body weight was observed with $4 \mathrm{~V}$ infusion of tofogliflozin (figure 3D). Taken together, these results indicate that the hyperphagic effect is strongest in the LV, followed by $3 \mathrm{~V}$, while no effect (or a mildly suppressive effect) on food intake was observed with administration into the $4 \mathrm{~V}$.

\section{Systemic administration of liraglutide attenuated the hyperphagic effect of ICV tofogliflozin}

Liraglutide is a GLP-1 receptor agonist that can increase satiety. ${ }^{24}$ To determine whether systemic administration of a GLP-1 receptor agonist can block the hyperphagic 


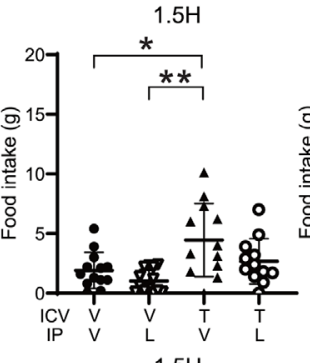

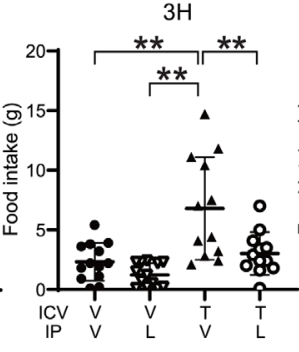

$3 \mathrm{H}$

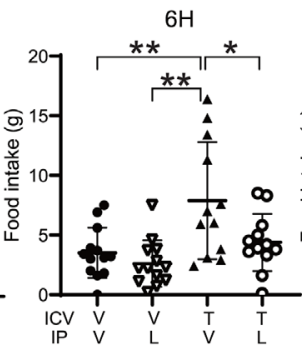

$6 \mathrm{H}$
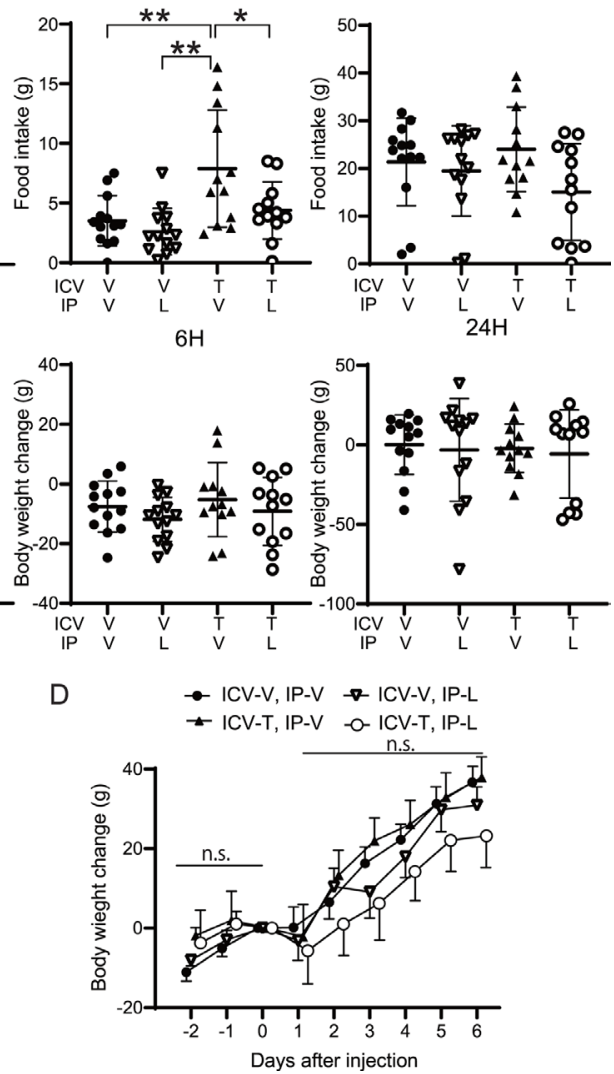

Figure 4 Effects of systemic liraglutide administration with ICV tofogliflozin on food intake. $0.52 \mu \mathrm{mol} / 3 \mu \mathrm{L}$ of tofogliflozin or vehicle was infused into the LV of rats, and $20 \mu \mathrm{g}$ of liraglutide was intraperitoneally injected daily from day 0 to day 6 . Food intake was measured at 1.5, 3, 6, and 24 hours after ICV infusion, and from 2 days before to 6 days after. (A) Cumulative food intake after ICV administration. (B) Body weight changes throughout time after ICV administration. (C) Daily food intake from 2 days before to 6 days after administration. (D) Changes in body weight after drug administration. (A,B) Data are presented as the mean $\pm S D$. Significance relative to vehicle control: ${ }^{*} p<0.05$, ${ }^{* *} p<0.01$. (C,D) Data are presented as the mean $\pm S E M$. Significance between IP vehicle vs IP liraglutide: ${ }^{* \star *} p<0.001$. Significance of interaction between IP liraglutide vs ICV tofogliflozin $\dagger p<0.05$. V, vehicle; T, tofogliflozin; L, liraglutide ICV-V+IPV, ICV-V+IPL, ICV-T+IPV, ICV-T+IP-L: $n=13,13,12$, and 12 , respectively. ICV, intracerebroventricular administration; LV, lateral ventricle.

effect of ICV SGLT2 inhibitors, we intraperitoneally injected $20 \mu \mathrm{mol}$ of liraglutide once a day into the rats treated with bolus ICV tofogliflozin. Consistent with previous experiments, the group receiving ICV tofogliflozin with intraperitoneal vehicle showed increased food intake compared with the ICV vehicle group (figure 4A). No significant changes in body weight were observed (figure 4B). Intraperitoneal injection of liraglutide significantly suppressed the hyperphagic effect of ICV tofogliflozin at 3 and 6 hours after treatment (figure 4A). In a three-way repeated measures ANOVA, IP liraglutide independently and significantly suppressed food intake for 5 days, with significant interaction between IP liraglutide and ICV tofogliflozin (figure 4C). No significant differences in body weight were observed (figure 4D).

\section{Changes in molecular signals in hypothalamic nuclei}

We conducted qPCR of c-fos expression in the hypothalamus at 1.5 hours after ICV administration of tofogliflozin. We found that c-fos, a marker of neural activity, was significantly upregulated in the ARC and tended to be increased in the PVH (figure 5A). Western blotting showed a significant increase in c-fos expression in the $\mathrm{LH}$, and a trend toward increased expression in the $\mathrm{PVH}$ (figure 5B). AMPK phosphorylation was examined in the LH (the feeding center), VMH (the satiety center), ARC, and PVH. There was a significant increase in AMPK phosphorylation in the $\mathrm{LH}$, and a trend toward decreased AMPK phosphorylation in the VMH (figure 5C). No significant changes in AMPK phosphorylation levels were observed in the ARC and PVH (figure 5C). Moreover, NPY and AgRP levels showed a decreasing trend with tofogliflozin, whereas in those of POMC did not significantly change (figure 5D).

\section{DISCUSSION}

We found that bolus administration of tofogliflozin to the ICV increases food intake for 5 days, whereas intraperitoneal injection does not. Thus, we consider the effects of ICV infusion attributable to direct actions within the CNS rather than due to leakage into the bloodstream. All three SGLT2 inhibitors induced hyperphagia when administered to the CNS. In addition, dapagliflozin showed a 

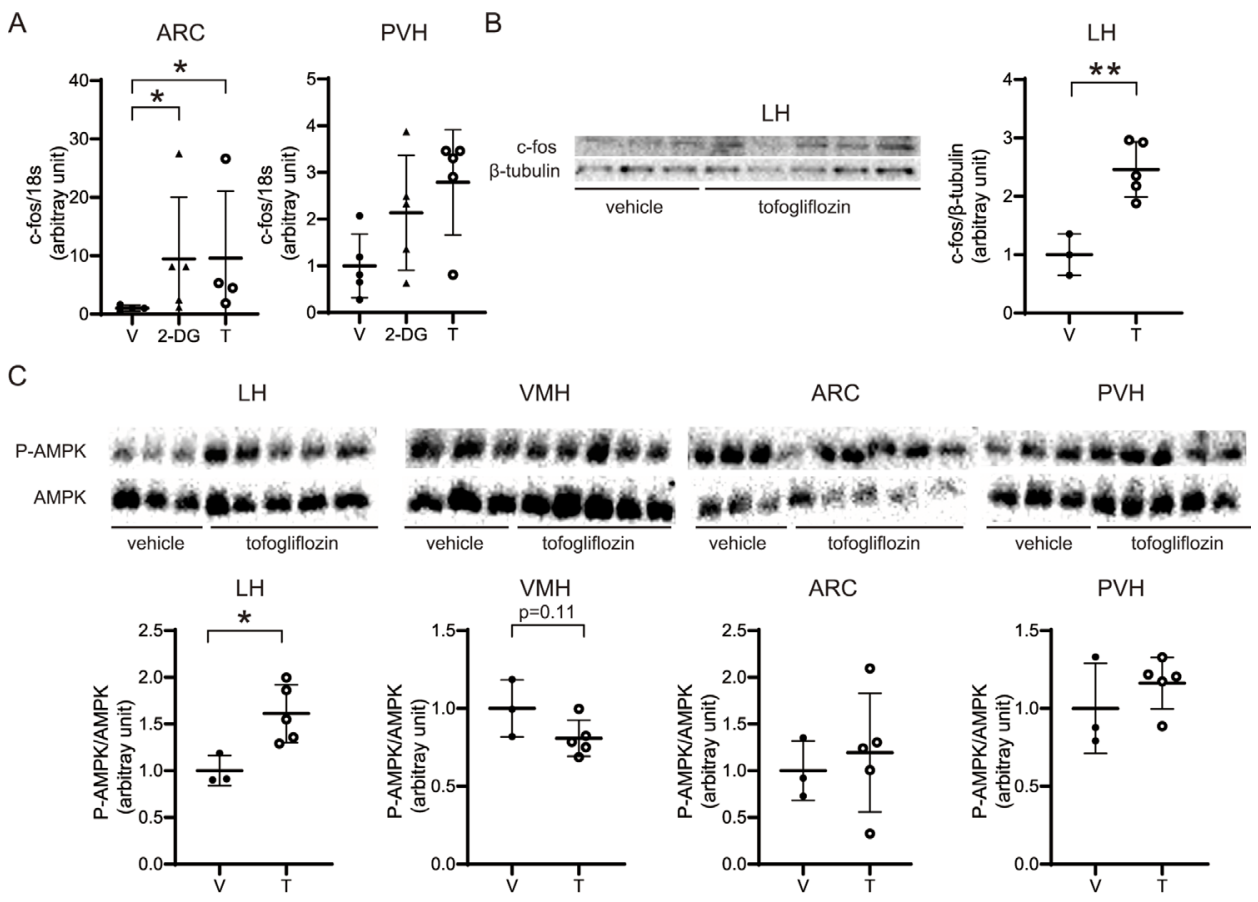

D
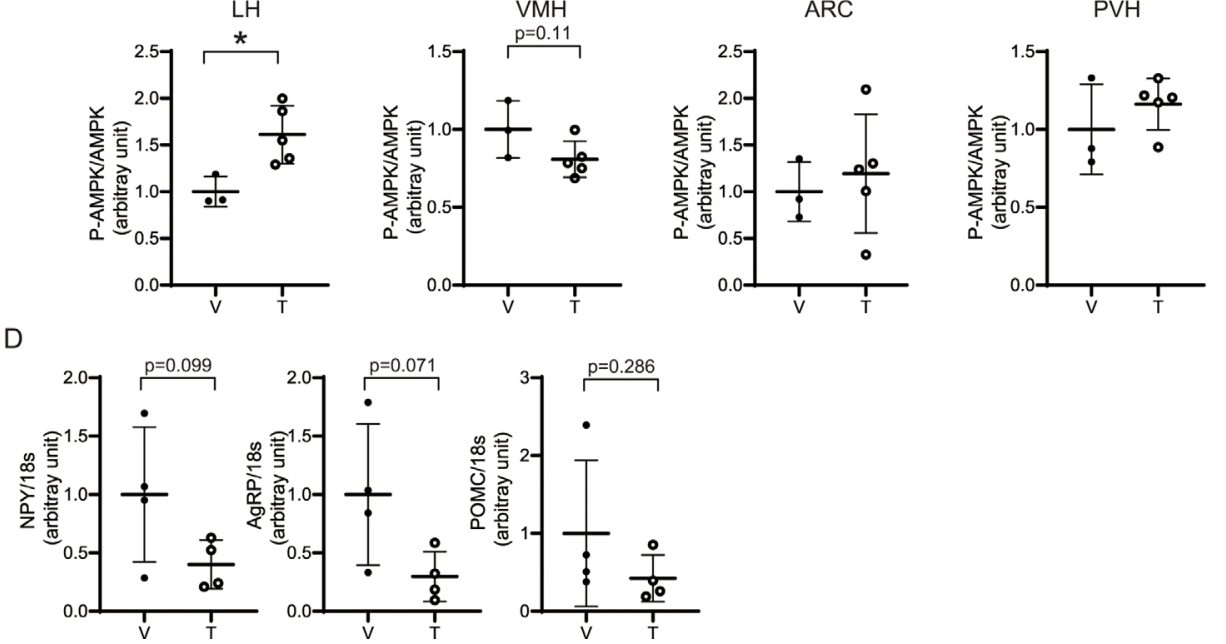

Figure 5 ICV of tofogliflozin altered c-fos expression and AMPK phosphorylation. At 1.5 hours after ICV infusion of 0.52 $\mu \mathrm{mol} / 3 \mu \mathrm{L}$ of tofogliflozin or vehicle into the LV, tissue samples were collected. (A) Expression of c-fos in the ARC and the PVH was assessed by RT-PCR and normalized to the expression of $18 \mathrm{~s}$ ribosomal RNA. (B) c-fos and $\beta$-tubulin expression were evaluated by immunoblotting. The results are shown as representative bands. All bands were quantified by densitometry. Band intensities of c-fos were normalized to $\beta$-tubulin. (C) Expression levels of total AMPK and AMPK phosphorylated at Thr172 were evaluated by immunoblotting. Results are shown as representative bands, and band are quantified by densitometry. Band intensities of phospho-AMPK were normalized to those of AMPK. (D) Expression of NPY, AgRP, and POMC in the ARC was assessed by RT-PCR and normalized to the expression of $18 \mathrm{~s}$ ribosomal RNA. Data are presented as the mean \pm SD. Significance relative to vehicle control: ${ }^{\star} \mathrm{p}<0.05,{ }^{\star *} \mathrm{p}<0.01$. V: vehicle, 2-DG: 2-deoxy-D-glucose, T: tofogliflozin A: (T, 2-DG, (V) $n=4,5$, and 4, respectively. B and C: (V, T): $n=3$ and 5, respectively. D: (V, T) n=4 and 4, respectively. AgRP, agouti-related protein; ARC, arcuate nucleus; ICV, intracerebroventricular administration; LH, lateral hypothalamus; LV, lateral ventricle; NPY, neuropeptide Y; POMC, pro-opiomelanocortin; PVH, paraventricular nucleus; VMH, ventromedial hypothalamic.

dose-dependent effect on food intake. The effect of ICV tofogliflozin was the strongest in the LV, while less or no effects in the $3 \mathrm{~V}$ or $4 \mathrm{~V}$, respectively. ICV infusion of SGLT2 inhibitors activated AMPK in the LH, and upregulated c-fos in the ARC and LH. The hyperphagic effect of tofogliflozin was blocked by systemic administration of liraglutide.

In several previous reports, oral administration of SGLT2 inhibitors was credited with increasing food intake in rats. ${ }^{25-29}$ In rats with diet-induced obesity, chronic dapagliflozin administration caused compensatory food intake and more than three times the weight loss in rats with paired food consumption. ${ }^{29}$ In a human study, a dissociation between expected and actual weight loss of approximately $8 \mathrm{~kg}$ was observed. ${ }^{4}$ In another study, steady state energy intake in the canagliflozin-treated subjects was calculated to have increased by $\sim 350 \mathrm{kcal} /$ day. ${ }^{30}$ To our knowledge, no research has focused on whether this hyperphagic effect is exerted via the CNS. Our observations in the present study raise the possibility that the clinically observed hyperphagic effects associated with SGLT2 inhibitors are, at least partially, exerted via the CNS. If true, this would imply that reduced transfer of SGLT2 inhibitors to the CNS would result in more effective weight loss.

In the present study, tofogliflozin induced a greater increase in food intake than did 2-DG or phlorizin. Several studies have reported that ICV phlorizin increases food intake. ${ }^{14-17}$ One showed that $0.26 \mu$ mol ICV phlorizin induced a stronger hyperphagic effect than 0.13 $\mu \mathrm{mol}$, while the effects of $0.26 \mu \mathrm{mol}$ and $0.52 \mu \mathrm{mol}$ were similar. ${ }^{17}$ Based on this report, we compared $0.52 \mu \mathrm{mol}$ 
of phlorizin with the same molar amount of tofogliflozin and found that the latter had a stronger effect on food intake. Two other previous studies demonstrated that approximately $20 \mu \mathrm{mol}$ ICV 2-DG induced a significant hyperphagic effect. ${ }^{1531}$ We thus employed 20 umol 2-DG, a dose considered sufficient to increase food intake. This amount of 2-DG tended to induce a hyperphagic effect in the present study, but its effect was smaller than that of tofogliflozin. Therefore, tofogliflozin was more effective in increasing food intake than phlorizin and 2-DG. These observations suggest that higher SGLT2/SLC5A2 selectivity contributes to enhanced food intake, supporting the presence of SGLT2/SLC5A2 in the rat CNS. The function of SGLT2/SLC5A2 itself might be associated with food intake.

The hyperphagic effect seems to be common to SGLT2 inhibitors, because all three SGLT2 inhibitors we used significantly increased food intake. There was, however, a difference in the degree of hyperphagia among groups given the same molar amount of each drug. SGLT2/ SLC5A2 selectivity differs among these drugs,${ }^{32}$ possibly contributing to this difference. SGLT1/SLC5A1 and SGLT3/SLC5A4 are present in the rat hypothalamus as glucose sensors. ${ }^{33}$ In humans, tofogliflozin is known to be less selective for SGLT3 versus SGLT2 than the other two SGLT2 inhibitors. ${ }^{32}$ Taken together with reports showing that SGLT3/SLC5A4 functions as a glucose sensor in the CNS ${ }^{34}$ additional effects on SGLT3/SLC5A4 may explain the stronger effects of tofogliflozin. Mice and rats have two isoforms of SGLT3/SLC5A4, unlike humans who have only one isoform. ${ }^{35}$ It is thus difficult to extrapolate findings from rats to humans. ${ }^{35}$ In addition, selectivity of SGLT2 inhibitors for SGLT2/SLC5A2 compared with SGLT1/SLC5A1 is reportedly lower in rats than in humans. ${ }^{36}$ Therefore, if the hyperphagic effect of SGLT2 inhibitors is SGLT2/SLC5A2-specific, it might be stronger in humans. Another possible reason is that differences in the excretion of SGLT2 inhibitors from the CNS may contribute to changes in hyperphagia. However, to the best of our knowledge, no studies have reported the rate or mechanism of SGLT2 inhibitors excretion in the CNS. Future experiments using ICV-radiolabelled SGLT2 inhibitors may clarify why ICV tofogliflozin had the strongest effect on food intake.

We attempted to identify brain regions responsible for the hyperphagic effect induced by SGLT2 inhibitors. ICV tofogliflozin infusion into the $3 \mathrm{~V}$ caused a milder increase in food intake than that into the LV. ICV tofogliflozin into the $4 \mathrm{~V}$ produced no significant hyperphagic effect. Conversely, body weight tended to be reduced by 4V-ICV administration. These results are in contrast to those of previous studies using phlorizin and 5-thioglucose. ${ }^{16} 1737$ ICV phlorizin in both the $\mathrm{LV}$ and $4 \mathrm{~V}$ reportedly induced a similar hyperphagic effect. ${ }^{17}$ In another report, ICV infusion of 5-thioglucose into both the $\mathrm{LV}$ and the $4 \mathrm{~V}$ increased food intake, and this effect was abolished only in the $\mathrm{LV}$ after obstruction of the cerebral aqueduct. ${ }^{37}$ According to one report, SGLT2/SLC5A2 was detected in the hypothalamus, amygdala, periaqueductal gray, and the nucleus of the solitary tract by immunohistochemical analysis. ${ }^{12}$ Another report showed that SGLT2/SLC5A2 is present in ependymal cells and choroid plexus epithelial cells. ${ }^{11}$ Thus, there is still no consensus regarding which CNS locations express SGLT2/SLC5A2. GLUTs are the primary transporter of glucose uptake in the CNS, and the contribution of SGLTs is low. ${ }^{38}$ Although differing in selectivity, tofogliflozin and phlorizin caused completely different effects when administered 4V-ICV, despite being SGLT inhibitors. These results suggest that the tofogliflozin target exists mainly in the forebrain, suggesting that SGLT2/SLC5A2, as a target of tofogliflozin, is present in the forebrain. This assumption is consistent with the sites previously suggested to harbor SGLT2/ SLC5A2. ${ }^{12}$ Another possibility is that spinal fluid flows from the LV to the lower ventricles, and the SGLT2 inhibitor thereby reaches more SGLT2-expressing ependymal cells expressed around the cerebral ventricle, which may explain why the effect is stronger in the upper ventricles.

In the present study, ICV administration of tofogliflozin increased c-fos expression and tended to suppress AgRP and NPY, without significant changes in POMC in the ARC. c-fos belongs to the Fos family, ${ }^{39}$ and various stimuli are known to selectively induce its expression. ${ }^{40}{ }^{41}$ Oral administration of SGLT2 inhibitors has been reported to enhance c-fos expression in the PVH, ARC, and LH, consistent with our observations. ${ }^{12}$ AMPK is known to be the main sensor of cellular energy status, and coordinates metabolism at the whole-body level. ${ }^{42}$ In the hypothalamus, regulation of AMPK phosphorylation is a mechanism for detecting nutritional changes. ${ }^{33} 43$ Early researchers referred to the $\mathrm{LH}$ and VMH as feeding and satiety centers, respectively. ${ }^{44}$ More recently, the LH has been suggested to be a core node within a distributed feeding network that integrates different feeding drives and motivates behaviour. ${ }^{45}$ The present study showed that ICV infusion of tofogliflozin activated AMPK in the $\mathrm{LH}$, consistent with a previous report that showed that systemic hypoglycemia could induce AMPK phosphorylation in the LH. ${ }^{46}$ These observations suggest that SGLT2/ SLC5A2 in the CNS directly or indirectly regulates feeding via AMPK phosphorylation in the LH. It was reported that the ARC projects to the $\mathrm{LH},{ }^{47}$ which may play an important role in feeding. ${ }^{48}$ Taken together, it is possible that tofogliflozin acts on the LH to enhance feeding, which in turn may induce compensatory suppression of AgRP and NPY expression. Systemic administration of exendin-4, a GLP-1 receptor agonist, has been reported to decrease AMPK phosphorylation in the $\mathrm{LH}^{49}$ In the present study, systemic injection of liraglutide blocked ICV infused tofogliflozin-mediated hyperphagia. One possible mechanism involves inhibition by GLP-1 of ICV administered SGLT2 inhibitor driven AMPK phosphorylation in the LH. Our findings may further clarify the role of the LH in food intake. Further studies using ICV-administrated AMPK inhibitors (ie, SBI-0206965) or allosteric activators (ie, A-769662, MK-8722, or 
PF-06409577) with tofogliflozin are necessary to further demonstrate that SGLT2 inhibitors enhance feeding via AMPK phosphorylation in the LH. The combination of SGLT2 inhibitors and GLP-1 receptor agonists reportedly results in greater weight loss than the use of a single agent in humans. ${ }^{50}$ Our results may provide a basic research perspective impetus for investigating the usefulness of combining SGLT2 inhibitors and GLP-1 receptor agonists.

The present study has several limitations. We did not prove that SGLT2/SLC5A2 regulates food intake. To determine the function of SGLT2/SLC5A2 in the brain, it is necessary to conduct experiments using knockout models. However, administering an SGLT2 inhibitor into the LV dramatically enhanced food intake, suggesting that the SGLT2 inhibitor acts directly in the CNS to induce hyperphagia. Another limitation is the dose of SGLT2 inhibitor administered to the CNS in this experiment, which would be higher than that of clinical use. To our knowledge, it is not known how much of the orally administered SGLT2 inhibitors could be transferred to the CNS. Thus, in addition to the acute and chronic effects on the CNS, as shown in this study, chronic energy loss via the excretion of urinary glucose could also induce compensatory food intake.

In summary, our study suggests that direct ICV administration of an SGLT2 inhibitor into the CNS increases food intake via phosphorylation of AMPK in the LH. While the physiological function of SGLT2 in the CNS has not been confirmed, our results support the theory that CNS SGLT2/SLC5A2 plays a regulatory role in food intake. This result may explain the increased food intake observed with the use of SGLT2 inhibitors in clinical practice. If we can develop SGLT2 inhibitors with lower or absent BBB penetration, diabetic treatment with reduced compensatory food intake might become possible.

Acknowledgements We would like to thank Dr Bierta Barfod and Editage (www. editage.jp) for English language editing. HOn is the guarantor of this work and, as such, had full access to all of the data in the study and takes responsibility for the integrity of the data and the accuracy of the data analysis.

Contributors KT, HOn, and KY designed the study. KT and HOn wrote the study protocol, performed the study, and wrote the manuscript. KT, HOc, AM, TO, JK, HY, and $\mathrm{HOn}$ contributed reagents/materials/analysis tools. All authors critically edited the manuscript and approved the final version.

Funding This work was supported by the grants JSPS KAKENHI 15 K09398 and $18 \mathrm{~K} 08502$ to $\mathrm{HOn}$ as well as $20 \mathrm{H} 00524$ to $\mathrm{KY}$ and by the Project for Elucidating and Controlling Mechanisms of Aging and Longevity of AMED 17gm5010002h0001, $18 \mathrm{gm} 5010002 \mathrm{~h} 0002,19 \mathrm{gm} 5010002 \mathrm{~h} 0003$, and 20gm5010002h0004 to KY.

Competing interests None declared.

Patient consent for publication Not required.

Ethics approval All experimental procedures were performed in accordance with specified guidelines and regulations for the care and use of laboratory animals. The Chiba University Institutional Animal Care and Use Committee approved all animal procedures.

Provenance and peer review Not commissioned; externally peer reviewed.

Data availability statement Data are available on reasonable request.

Supplemental material This content has been supplied by the author(s). It has not been vetted by BMJ Publishing Group Limited (BMJ) and may not have been peer-reviewed. Any opinions or recommendations discussed are solely those of the author(s) and are not endorsed by BMJ. BMJ disclaims all liability and responsibility arising from any reliance placed on the content. Where the content includes any translated material, BMJ does not warrant the accuracy and reliability of the translations (including but not limited to local regulations, clinical guidelines, terminology, drug names and drug dosages), and is not responsible for any error and/or omissions arising from translation and adaptation or otherwise.

Open access This is an open access article distributed in accordance with the Creative Commons Attribution Non Commercial (CC BY-NC 4.0) license, which permits others to distribute, remix, adapt, build upon this work non-commercially, and license their derivative works on different terms, provided the original work is properly cited, appropriate credit is given, any changes made indicated, and the use is non-commercial. See: http://creativecommons.org/licenses/by-nc/4.0/.

\section{ORCID iDs}

Kenji Takeda http://orcid.org/0000-0001-8323-2488 Hiraku Ono http://orcid.org/0000-0002-3550-9339

Ko Ishikawa http://orcid.org/0000-0001-5788-2217

Yoshiro Maezawa http://orcid.org/0000-0001-5567-9901

\section{REFERENCES}

1 Mueckler M, Thorens B. The SLC2 (GLUT) family of membrane transporters. Mol Aspects Med 2013:34:121-38.

2 Deng D, Yan N. Glut, SGLT, and sweet: structural and mechanistic investigations of the glucose transporters. Protein Sci 2016;25:546-58.

3 Navale AM, Paranjape AN. Glucose transporters: physiological and pathological roles. Biophys Rev 2016;8:5-9.

4 Ferrannini G, Hach T, Crowe S, et al. Energy balance after sodiumglucose cotransporter 2 inhibition. Diabetes Care 2015;38:1730-5.

5 Chen J, Williams S, Ho S, et al. Quantitative PCR tissue expression profiling of the human SGLT2 gene and related family members. Diabetes Ther 2010;1:57-92.

6 Vallon V, Platt KA, Cunard R, et al. Sglt2 mediates glucose reabsorption in the early proximal tubule. J Am Soc Nephrol 2011;22:104-12.

7 Vrhovac I, Balen Eror D, Klessen D, et al. Localizations of Na+-Dglucose cotransporters SGLT1 and SGLT2 in human kidney and of SGLT1 in human small intestine, liver, lung, and heart. Pflugers Arch - Eur J Physiol 2015;467:1881-98.

8 Wright EM, Loo DDF, Hirayama BA. Biology of human sodium glucose transporters. Physiol Rev 2011;91:733-94.

9 Zhou L, Cryan EV, D'Andrea MR, et al. Human cardiomyocytes express high level of $\mathrm{Na}$ +/glucose cotransporter 1 (SGLT1). J Cell Biochem 2003;90:339-46.

10 Oerter S, Förster C, Bohnert M. Validation of sodium/glucose cotransporter proteins in human brain as a potential marker for temporal narrowing of the trauma formation. Int $J$ Legal Med 2019;133:1107-14

11 Chiba Y, Sugiyama Y, Nishi N, et al. Sodium/glucose cotransporter 2 is expressed in choroid plexus epithelial cells and ependymal cells in human and mouse brains. Neuropathology 2020;40:482-91.

12 Nguyen T, Wen S, Gong M, et al. Dapagliflozin activates neurons in the central nervous system and regulates cardiovascular activity by inhibiting SGLT-2 in mice. Diabetes Metab Syndr Obes 2020;13:2781-99.

13 Yu AS, Hirayama BA, Timbol G, et al. Functional expression of SGLTs in rat brain. Am J Physiol Cell Physiol 2010;299:C1277-84

14 Glick Z, Mayer J. Hyperphagia caused by cerebral ventricular infusion of phloridzin. Nature 1968;219:1374.

15 Tsujii S, Bray GA. Effects of glucose, 2-deoxyglucose, phlorizin, and insulin on food intake of lean and fatty rats. Am J Physiol 1990;258:E476-81.

16 Flynn FW, Grill HJ. Fourth ventricular phlorizin dissociates feeding from hyperglycemia in rats. Brain Res 1985;341:331-6.

17 Li A-J, Wang Q, Dinh TT, et al. Stimulation of feeding by three different glucose-sensing mechanisms requires hindbrain catecholamine neurons. Am J Physiol Regul Integr Comp Physiol 2014;306:R257-64.

18 Gonzàlez JA, Reimann F, Burdakov D. Dissociation between sensing and metabolism of glucose in sugar sensing neurones. J Physiol 2009;587:41-8.

19 Pénicaud L, Thompson DA. Effects of systemic or intracerebroventricular naloxone injection on basal and 2-deoxy-Dglucose-induced ingestive behavior. Life Sci 1984;35:2297-302.

20 Smith GP, Epstein AN. Increased feeding in response to decreased glucose utilization in the rat and monkey. Am J Physiol 1969;217:1083-7. 
21 Ferrannini E, Co-transporters S-G. Sodium-Glucose co-transporters and their inhibition: clinical physiology. Cell Metab 2017;26:27-38.

22 Ann S, Pellegrino PLJ, Cushman AJ. A stereotaxic atlas of the rat brain. second edition. PLENUM PUBLISHING CORPORATION, 1979.

23 Sumita T, Ono H, Suzuki T, et al. Mediobasal hypothalamic PTEN modulates hepatic insulin resistance independently of food intake in rats. Am J Physiol Endocrinol Metab 2014;307:E47-60.

24 Trujillo JM, Nuffer W, Ellis SL. GLP-1 receptor agonists: a review of head-to-head clinical studies. Ther Adv Endocrinol Metab 2015;6:19-28.

25 Suzuki M, Takeda M, Kito A, et al. Tofogliflozin, a sodium/glucose cotransporter 2 inhibitor, attenuates body weight gain and fat accumulation in diabetic and obese animal models. Nutr Diabetes 2014;4:e125.

26 Iuchi H, Sakamoto M, Matsutani D, et al. Time-dependent effects of ipragliflozin on behaviour and energy homeostasis in normal and type 2 diabetic rats: continuous glucose telemetry analysis. Sci Rep 2017;7:11906.

27 Kojima N, Williams JM, Takahashi T, et al. Effects of a new SGLT2 inhibitor, luseogliflozin, on diabetic nephropathy in T2DN rats. $J$ Pharmacol Exp Ther 2013;345:464-72.

28 Vickers SP, Cheetham SC, Headland KR, et al. Combination of the sodium-glucose cotransporter-2 inhibitor empagliflozin with orlistat or sibutramine further improves the body-weight reduction and glucose homeostasis of obese rats fed a cafeteria diet. Diabetes Metab Syndr Obes 2014;7:265-75.

29 Devenny JJ, Godonis HE, Harvey SJ, et al. Weight loss induced by chronic dapagliflozin treatment is attenuated by compensatory hyperphagia in diet-induced obese (DIO) rats. Obesity 2012;20:1645-52.

30 Polidori D, Sanghvi A, Seeley RJ, et al. How strongly does appetite counter weight loss? quantification of the feedback control of human energy intake. Obesity 2016;24:2289-95.

31 Miselis RR, Epstein AN. Feeding induced by intracerebroventricular 2-deoxy-D-glucose in the rat. Am J Physiol 1975;229:1438-47.

32 Suzuki M, Honda K, Fukazawa M, et al. Tofogliflozin, a potent and highly specific sodium/glucose cotransporter 2 inhibitor, improves glycemic control in diabetic rats and mice. J Pharmacol Exp Ther 2012;341:692-701.

33 Mountjoy PD, Rutter GA. Glucose sensing by hypothalamic neurones and pancreatic islet cells: AMPle evidence for common mechanisms? Exp Physiol 2007;92:311-9.

34 Díez-Sampedro A, Hirayama BA, Osswald C, et al. A glucose sensor hiding in a family of transporters. Proc Natl Acad Sci U S A 2003;100:11753-8.

35 Soták M, Marks J, Unwin RJ. Putative tissue location and function of the SLC5 family member SGLT3. Exp Physiol 2017;102:5-13.
36 Grempler R, Thomas L, Eckhardt M, et al. Empagliflozin, a novel selective sodium glucose cotransporter-2 (SGLT-2) inhibitor: characterisation and comparison with other SGLT-2 inhibitors. Diabetes Obes Metab 2012;14:83-90.

37 Ritter RC, Slusser PG, Stone S. Glucoreceptors controlling feeding and blood glucose: location in the hindbrain. Science 1981;213:451-2.

38 Sala-Rabanal M, Hirayama BA, Ghezzi C, et al. Revisiting the physiological roles of SGLTs and GLUTs using positron emission tomography in mice. J Physiol 2016;594:4425-38.

39 Milde-Langosch K. The Fos family of transcription factors and their role in tumourigenesis. Eur J Cancer 2005;41:2449-61.

40 Sagar SM, Sharp FR, Curran T. Expression of c-fos protein in brain: metabolic mapping at the cellular level. Science 1988;240:1328-31.

41 Morgan JI, Curran T. Stimulus-transcription coupling in neurons: role of cellular immediate-early genes. Trends Neurosci 1989;12:459-62.

42 Garcia D, Shaw RJ. Ampk: mechanisms of cellular energy sensing and restoration of metabolic balance. Mol Cell 2017;66:789-800.

43 Minokoshi Y, Alquier T, Furukawa N, et al. Amp-Kinase regulates food intake by responding to hormonal and nutrient signals in the hypothalamus. Nature 2004;428:569-74.

44 Anand BK, Brobeck JR. Hypothalamic control of food intake in rats and cats. Yale J Biol Med 1951;24:123-40.

45 Petrovich GD. Lateral hypothalamus as a Motivation-Cognition interface in the control of feeding behavior. Front Syst Neurosci 2018;12:14.

46 Alhamami HN, Uddin MM, Mahmood ASMH, et al. Lateral but not medial hypothalamic AMPK activation occurs at the hypoglycemic nadir in Insulin-injected male rats: impact of caudal dorsomedial hindbrain catecholamine signaling. Neuroscience 2018;379:103-14.

47 Elias CF, Aschkenasi C, Lee C, et al. Leptin differentially regulates NPY and POMC neurons projecting to the lateral hypothalamic area. Neuron 1999;23:775-86.

48 Elias CF, Saper CB, Maratos-Flier E, et al. Chemically defined projections linking the mediobasal hypothalamus and the lateral hypothalamic area. J Comp Neurol 1998;402:442-59.

49 Hurtado-Carneiro V, Sanz C, Roncero I, et al. Glucagon-Like peptide 1 (GLP-1) can reverse AMP-activated protein kinase (AMPK) and S6 kinase (p70S6K) activities induced by fluctuations in glucose levels in hypothalamic areas involved in feeding behaviour. Mol Neurobiol 2012;45:348-61.

50 Frías JP, Guja C, Hardy E, et al. Exenatide once Weekly plus dapagliflozin once daily versus exenatide or dapagliflozin alone in patients with type 2 diabetes inadequately controlled with metformin monotherapy (DURATION-8): a 28 week, multicentre, double-blind, phase 3, randomised controlled trial. Lancet Diabetes Endocrinol 2016;4:1004-16. 